\title{
A metabolomic study on the responses of daphnia magna exposed to silver nitrate and coated silver nanoparticles
}

\author{
LianZhen $\mathrm{Li}^{\mathrm{a}}$, Huifeng $\mathrm{Wu}^{\mathrm{a}, *}$, Chenglong $\mathrm{Ji}^{\mathrm{a}}$, Cornelis A.M. van Gestel ${ }^{\mathrm{b}}$, Herbert E. Allen ${ }^{\mathrm{c}}$, \\ Willie J.G.M. Peijnenburg ${ }^{\mathrm{d}, \mathrm{e}}$ \\ ${ }^{a}$ Key Laboratory of Coastal Environmental Processes and Ecological Remediation, Yantai Institute of Coastal Zone Research (YIC), Chinese Academy of \\ Sciences (CAS), Shandong Provincial Key Laboratory of Coastal Environmental Processes, YICCAS, no.17, Chunhui Road, Yantai, Shandong 264003, PR China \\ ${ }^{\mathrm{b}}$ Department of Ecological Science, Faculty of Earth and Life Sciences, VU University, De Boelelaan 1085, 1081 HV Amsterdam, The Netherlands \\ ${ }^{\mathrm{c}}$ Department of Civil and Environmental Engineering, University of Delaware, Newark, DE 19716, USA \\ ${ }^{\mathrm{d}}$ National Institute of Public Health and the Environment, Center for Safety of Substances and Products, P.O. Box 1, 3720 BA Bilthoven, The Netherlands \\ e Institute of Environmental Sciences (CML), Leiden University, Leiden, The Netherlands
}

\section{A R T I C L E I N F O}

\section{Article history:}

Received 30 December 2014

Received in revised form

5 May 2015

Accepted 6 May 2015

Available online 14 May 2015

Keywords:

Daphnia magna

Silver nanoparticle

Metabolomics

Silver ion

Sublethal toxicity

\begin{abstract}
A B S T R A C T
We examined the short-term toxicity of AgNPs and $\mathrm{AgNO}_{3}$ to Daphnia magna at sublethal levels using ${ }^{1} \mathrm{H}$ NMR-based metabolomics. Two sizes of polyvinylpyrrolidone-coated AgNPs (10 and $40 \mathrm{~nm}$ ) were synthesized and characterized and their $\mathrm{Ag}^{+}$release was studied using centrifugal ultrafiltration and inductively coupled plasma mass spectrometry. Multivariate statistical analysis of the ${ }^{1} \mathrm{H}$ NMR spectra showed significant changes in the D. magna metabolic profiles following $48 \mathrm{~h}$ exposure to both AgNP particle sizes and $\mathrm{Ag}^{+}$exposure. Most of the metabolic biomarkers for AgNP exposure, including 3-hydroxybutyrate, arginine, lysine and phosphocholine, were identical to those of the $\mathrm{Ag}^{+}$-exposed groups, suggesting that the dominant effects of both AgNPs were due to released $\mathrm{Ag}^{+}$. The observed metabolic changes implied that the released $\mathrm{Ag}^{+}$induced disturbance in energy metabolism and oxidative stress, a proposed mechanism of AgNP toxicity. Elevated levels of lactate in all AgNP-treated but not in $\mathrm{Ag}^{+}$-treated groups provided evidence for $\mathrm{Ag}-\mathrm{NP}$ enhanced anaerobic metabolism. These findings show that ${ }^{1} \mathrm{H}$ NMR-based metabolomics provides a sensitive measure of $D$. magna response to AgNPs and that further targeted assays are needed to elucidate mechanisms of action of nanoparticle-induced toxicity.
\end{abstract}

(c) 2015 Elsevier Inc. All rights reserved.

\section{Introduction}

With the increased application of nanosized silver in consumer products, predominately as bactericide, an increasing quantity of silver nanoparticles (AgNPs) is finding its way into wastewater and hence to rivers and streams. In addition to being bactericidal, AgNPs are toxic to other taxa. To date, most of the available data on adverse effects of AgNPs involve freshwater species. The documented effects include developmental deformities in zebrafish (Massarsky et al., 2013), altered stress-related gene expressions in Japanese medaka (Chae et al., 2009), and respiratory stress in Eurasian perch (Bilberg et al., 2010).

Despite many recent publications on toxicological effects of AgNPs, it remains unclear whether observed toxicity is specifically related to nanoparticles or is due to the effects of dissolved forms of Ag released from AgNPs. Laban et al. (2010) stated that the mortality of Pimephales promelas embryos after $96 \mathrm{~h}$ exposure to AgNPs was not attributed solely to the released Ag ions but rather to the AgNPs themselves. Li et al. (2010) also showed that the active component in the toxicity testing of AgNPs to Daphnia magna was the reduced form of silver and not the presence of excess dissolved $\mathrm{Ag}^{+}$. It was also concluded that $\mathrm{Ag}$ had an enhanced acute toxicity to freshwater cladocerans, Ceriodaphnia dubia, if present as AgNPs compared to when present as the ionic form (Griffitt, et al., 2008). Other authors have published evidence that the toxicity of AgNPs can entirely be explained from co-occurring $\mathrm{Ag}^{+}$(Meyer et al., 2010; Kim et al., 2011). Using genetic analysis, Meyer et al. (2010) found evidence that the toxicity of polyvinylpyrrolidone (PVP) coated AgNPs to Caenorhabditis elegans was mediated by the release of ionic silver. Kim et al. (2011) also

\footnotetext{
* Corresponding author. Fax: +86535 2109000.

E-mail address: hfwu@yic.ac.cn (H. Wu).
} 
concluded that AgNP suspensions were not acutely toxic to $D$. magna when excluding $\mathrm{Ag}^{+}$with synthesized sorbents. Thus, there is an ongoing debate over whether the observed toxicity is due to the release of $\mathrm{Ag}^{+}$alone, or if the nanoparticles themselves exert a direct toxic effect.

For evaluation of the potential toxicity of nanoparticles, fast and high throughput methods are needed. The omics technologies are particularly well suited to evaluate toxicity in both in vitro and in vivo systems. Metabolomics can rapidly screen for biomarkers related to predefined pathways or processes in biofluids and tissues (Lin et al., 2006; Viant et al., 2003). Specifically, little is known about the mechanisms of action of nanoparticles which generally are difficult to measure by conventional methods. Thus, metabolomics can provide possible mechanistic insight into nanotoxicity. Metabolomics has been used to study the toxic effects of a wide variety of environmental contaminants to aquatic organisms (Bundy et al., 2009; Liu et al., 2011) and was recently applied to identify mechanisms of toxicity of AgNPs in rats (Hadrup et al., 2012) and titanium dioxide nanomaterial in earthworms (Whitfield Aslund et al., 2012). Yet, despite the cladoceran D. magna being one of the most widely utilized aquatic test species, few metabolomics studies have been reported, especially for nanomaterials. This is a knowledge gap that this research aims to redress.

D. magna is a freshwater invertebrate with many attributes that make it an ideal sentinel organism and it plays important ecological roles in freshwater habitats. It is among the most sensitive organisms used in ecotoxicology and a standard test organism for the standardized protocols of the U.S. Environmental Protection Agency (EPA), Organization for Economic Cooperation and Development (OECD), and International Standardization Organization (ISO) (Baun et al., 2008; OECD Guidelines test no. 202, 2004).

In this study, the metabolomics approach was applied to evaluate the potential toxicological effects of PVP-coated AgNPs at sublethal concentrations $(2 \mu \mathrm{g} / \mathrm{L}$ and $10 \mu \mathrm{g} \mathrm{Ag} / \mathrm{L})$, using D. magna as the test organism. An $\mathrm{AgNO}_{3}$ solution was used to compare the toxic effects of $\mathrm{Ag}^{+}$to those of the AgNPs. Using global analysis of metabolomics for the discovery of biomarkers, we sought to examine whether AgNP toxicity could be attributed to nanoparticles or to dissolved silver released from the AgNPs. The extent to which AgNP sizes modifies toxicity to $D$. magna was determined by using two types of size-controlled AgNPs.

\section{Materials and methods}

\subsection{Preparation and characterization of metal salt solutions and nanoparticle suspensions}

Silver nitrate $\left(\mathrm{AgNO}_{3}\right.$; ACS reagent, 99.0\%; Sigma-Aldrich) was used as a source of $\mathrm{Ag}^{+}$for toxicity testing. Two sizes of PVP coated AgNPs (10 and $40 \mathrm{~nm}$ ) were synthesized by reducing an $\mathrm{AgNO}_{3}$ solution, using sodium hypophosphite and stabilizing with PVP. Details of particle synthesis, purification and storage are described in detail by Liu et al. (2009) and are summarized in Supplementary information. The exact Ag concentrations of NPs solutions were measured using inductively coupled plasma-mass spectrometry (ICP-MS; Agilent 7500i, Agilent Technologies Co. Ltd, USA) after digestion with $65 \% \mathrm{HNO}_{3}$.

The morphology and size of the obtained AgNPs were determined by transmission electron microscopy (TEM) (H-7500, Hitachi, Japan) at $80 \mathrm{kV}$ accelerated voltage on a Philips EM420 at $120 \mathrm{kV}$ and scanning electron microscopy (SEM) (S-4800, Hitachi, Japan) coupled with an energy dispersive X-ray spectroscopy (EDS) (Oxford, UK) at $15 \mathrm{kV}$. TEM samples were prepared by placing a drop of fresh AgNP suspension in water on a copper grid with a continuous carbon film coating, followed by solvent evaporation overnight at room temperature. The size distribution of the nanoparticles was estimated using Image-Pro plus software (Media Cybernetics). At least 300 particles were counted from a multipicture in each case. The UV-vis spectra at 300-700 nm were obtained using a Shimadzu UV-1700 PharmaSpec. AgNPs were characterized in de-ionized water and exposure medium under experimental conditions for size and Zeta potential by Dynamic Light Scattering (DLS) using a Zeta Sizer (Nano ZS, Malvern Instruments). Three successive measurements within an interval of two minutes were performed on two samples.

The release of soluble $\mathrm{Ag}$ at low $(2 \mu \mathrm{g} \mathrm{Ag} / \mathrm{L})$ and high $(10 \mu \mathrm{g} \mathrm{Ag} /$ L) AgNP concentrations in reconstituted water (RW, see below) was determined at the end of the exposures by ultracentrifugation through a $3 \mathrm{kDa}$ membrane (pore size around $1 \mathrm{~nm}$, Millipore, USA) as described by Navarro et al. (2008).

\subsection{Animals and exposure study}

D. magna were kindly provided by Dr. Qiaoguo Tan (Xiamen University, P.R. China). The RW water used had a pH of 7.2, hardness of $73 \mathrm{mg} / \mathrm{L}$ as $\mathrm{CaCO}_{3}$,containing $24 \mathrm{mg} / \mathrm{L} \mathrm{Ca}, 3.1 \mathrm{mg} / \mathrm{L} \mathrm{Mg}$, $2.1 \mathrm{mg} / \mathrm{L}$ of $\mathrm{K}$ and $26.3 \mathrm{mg} / \mathrm{L}$ of $\mathrm{Na}$. It was prepared from the following analytical reagent-grade chemicals: Sodium bicarbonate, calcium sulfate, magnesium sulfate, potassium nitrate (Sigma-Aldrich, Shanghai, China) added to purified Milli-Qt water (Milli-Q, Millipore, Bedford, MA, USA, $18.2 \mathrm{M} \Omega \mathrm{cm}$ ). To avoid an uncontrolled loss of silver ions from RW caused by precipitation as $\mathrm{AgCl}$, we used a specially designed chloride free RW in this study. At least $10 \mathrm{~mL}$ of water was allocated to each individual D. magna and refreshed every 2 days. The freshwater green alga, Chlamydomonas reinhardtii, was offered as food daily at a dose of $0.5-1.0 \times 10^{6}$ cells per individual depending on the age of the animals. The routine culture and all experiments were conducted under a light/dark cycle of $14 \mathrm{~h}: 10 \mathrm{~h}$ and a temperature of $24^{\circ} \mathrm{C}$.

\subsection{Acute toxicity tests}

The acute $(48 \mathrm{~h})$ toxicity tests were conducted following methods described by Zhao and Wang (2012), see Supplementary information for more details. To compare the effects of AgNPs and the released $\mathrm{Ag}^{+}$ions on $D$. magna, two different experiments were performed. In the first experiment, the impact of NP size and concentration on AgNP toxicity was investigated. A series of preliminary experiments were conducted to determine the range of chemical concentrations that caused mortality of $D$. magna. Based on the determined concentration ranges, a low concentration of $2 \mu \mathrm{g} / \mathrm{L}$ and a high concentration of $10 \mu \mathrm{g} / \mathrm{L}$ were selected for the AgNP exposures. One hundred 10-day old individuals were placed in $1000 \mathrm{~mL}$ of RW containing a low or a high AgNP concentration for $48 \mathrm{~h}$ and media were refreshed every $24 \mathrm{~h}$ to maintain the NPs in suspension. Five replicates were used for each treatment. The animals were not fed during the test. To compare the difference in toxicity between $\mathrm{AgNPs}$ and $\mathrm{Ag}^{+}$, an $\mathrm{AgNO}_{3}$ standard solution $(1 \mathrm{~g}$ $\mathrm{Ag} / \mathrm{L}$ ) was used as a stock to prepare different $\mathrm{Ag}^{+}$concentrations in the second experiment. $\mathrm{AgNO}_{3}$ concentrations were chosen based on the concentration of soluble Ag released from AgNPs after $48 \mathrm{~h}$ under the experimental conditions. Four replicates per treatment were used in the experiment with approximately one hundred 10-day old D. magna in each replicate sample.

\subsection{Metabolite extraction}

For the metabolomics analyses, polar metabolites in each $D$. magna sample were extracted by the modified extraction protocol described previously (Wu et al., 2008). Briefly, each D. magna 
sample was homogenized and extracted in $4 \mathrm{ml} / \mathrm{g}$ of methanol, $5.25 \mathrm{ml} / \mathrm{g}$ of water and $2 \mathrm{ml} / \mathrm{g}$ of chloroform. The mixture was shaken and centrifuged ( $5 \mathrm{~min}, 3000 \mathrm{~g}$, at $4{ }^{\circ} \mathrm{C}$ ), and the supernatant was removed. A total of $2 \mathrm{mLg}^{-1}$ of chloroform and $2 \mathrm{~mL} \mathrm{~g}^{-1}$ of water was added to the supernatant, and the mixture was vortexed and then centrifuged again $\left(10 \mathrm{~min}, 3000 \mathrm{~g}, 4^{\circ} \mathrm{C}\right.$ ). The methanol/water layer with polar metabolites was transferred to a glass vial and dried in a centrifugal concentrator. The extracts were then re-suspended in $600 \mu \mathrm{L}$ phosphate buffer $(100 \mathrm{mM}$ $\mathrm{Na}_{2} \mathrm{HPO}_{4}$ and $\mathrm{NaH}_{2} \mathrm{PO}_{4}$, including $0.5 \mathrm{mM}$ TSP, pH 7.0) in $\mathrm{D}_{2} \mathrm{O}$. The mixture was vortexed and then centrifuged at $3000 \mathrm{~g}$ for $5 \mathrm{~min}$ at $4{ }^{\circ} \mathrm{C}$. The supernatant $(550 \mu \mathrm{L})$ was transferred into a $5 \mathrm{~mm} \mathrm{nu}-$ clear magnetic resonance (NMR) tube prior to NMR analysis.

\section{5. ${ }^{1} \mathrm{H}$ NMR Spectroscopy}

Metabolite extracts of $D$. magna were analyzed on a Bruker AV 500 NMR spectrometer performed at $500.18 \mathrm{MHz}$ (at $25^{\circ} \mathrm{C}$ ) as described previously by Liu et al. (2011) All ${ }^{1} \mathrm{H}$ NMR spectra were phased, baseline-corrected, and calibrated (TSP at $0.0 \mathrm{ppm}$ ) manually using TopSpin (version 2.1, Bruker).

\subsection{Spectral pre-processing and multivariate analysis}

All one dimensional ${ }^{1} \mathrm{H}$ NMR spectra were converted to a data matrix using the custom-written ProMetab software in Matlab version 7.0 (The MathsWorks, Natick, MA) (Parsons et al., 2007). Each spectrum was segmented into bins with a width of $0.005 \mathrm{ppm}$ between 0.2 and $10.0 \mathrm{ppm}$. The bins of the residual water peaks between 4.70 and $5.20 \mathrm{ppm}$ were excluded from the NMR spectra. The total spectral area of the remaining bins was normalized to unity to facilitate comparison between the spectra. All the NMR spectra were generalized log transformed (glog) with a transformation parameter $\lambda=2.0 \times 10^{-7}$ to stabilize the variance across the spectral bins and to increase the weightings of the less intense peaks (Parsons et al., 2007). Data were mean-centered before multivariate analysis.

Principal component analysis (PCA) was used to reduce the dimensionality of the data and summarize the similarities and differences between multiple NMR spectra (Liu et al., 2011). Oneway analysis of variance (ANOVA) was conducted on the PC scores from each group to test the statistical significance $(P<0.05)$ of separations. Furthermore, partial least squares discriminant analysis (PLS-DA) and orthogonal projection to latent structures with discriminant analysis (O-PLS-DA), were sequentially carried out to discover the significant metabolite variations related to exposures. The results were visualized in scores plots to show the classifications and corresponding loadings plots to show the NMR spectral variables contributing to the classifications. The model coefficients were calculated from the coefficients incorporating the weight of the variables to enhance interpretability of the model. Then metabolic differences contributing to the classifications between control and treatments could be detected in the coefficient-coded loadings plots. The coefficient plots were generated using MATLAB (V7.0, the Mathworks Inc., Natwick, USA) with an in-house developed program and were color-coded with absolute correlation coefficient $(r)$ values. A hot color (e.g., red) corresponds to the metabolites with highly positive or negative significances in discriminating between groups, while a cool color (e.g. blue) means no significance. The correlation coefficient was determined according to the test for significance of the Pearson's product-moment correlation coefficient. Validity of the model was tested using 10-fold cross validation and the cross-validation parameter $Q^{2}$ was calculated. An additional validation using a permutation test (permutation number $=200$ ) was also conducted to evaluate the validity of the PLS-DA models. The $R^{2}$ in the permuted plot described how well the data fitted the derived model, whereas $Q^{2}$ describes the predictive ability of the derived model and provides a measure of the model quality. If the maximum value of $Q^{2}\left(Q^{2} \max \right)$ from the permutation test was smaller than or equal to the $Q^{2}$ of the real model, the model was regarded as a predictable model. Similarly, the $R^{2}$ value and the difference between $R^{2}$ and $Q^{2}$ were used to evaluate the possibility of over-fitted models (Feng et al. 2011, 2013). Metabolites were assigned following the tabulated chemical shifts (Fan, 1996) and by using the Chenomx software (Evaluation Version, Chenomx Inc., Edmonton, Alberta, Canada).

\section{Results}

\subsection{AgNP characterization}

The SEM images of the PVP-coated AgNPs indicated spherical shapes of zerovalent silver, with mean size of $40 \pm 10$ and $10 \pm 4.0 \mathrm{~nm}$ (Fig. $1 \mathrm{~A}$ and $\mathrm{B}$ ). The SEM-EDS elemental spectrum showed the existence of $\mathrm{Ag}$, chemically confirming the formation of AgNP particles. These two types of AgNPs are referred to as smaller AgNPs (S AgNP) and larger AgNPs (L AgNP). TEM analysis confirmed these results, and also revealed that the size of the $\mathrm{L}$ AgNP ranged from 30 to $50 \mathrm{~nm}$ (Fig. 1C), while the S AgNP had an average size of $10 \mathrm{~nm}$ (Fig. 1D). A single plasmonic resonance peak was observed at $410 \mathrm{~nm}$ (Fig. S1, Supplementary information), which was indicative of the presence of AgNPs.

When dispersed in DI water, the hydrodynamic diameter of both the nanoparticles increased compared to their primary size, but the average diameter of the AgNPs remained under $60 \mathrm{~nm}$ in all cases. When the particles were dispersed in $\mathrm{RW}$ at $\mathrm{pH} 7.2$, their hydrodynamic diameter increased even further, and the mean size of the agglomerated particles in the test medium was 55.4 $( \pm 10.1) \mathrm{nm}$ and $89.3( \pm 21.5) \mathrm{nm}$ for S AgNP and L AgNP, respectively. The surface charge determined by measurement of their zeta-potential showed that the S AgNP had a more negative Zeta potential $(-33.6 \pm 8.25 \mathrm{mV})$ than the L AgNP $(-25.7 \pm 7.87 \mathrm{mV})$. Despite some aggregation in the RW, the average size of the NP agglomerates remained below $100 \mathrm{~nm}$ under these conditions and remained stable for at least $48 \mathrm{~h}$, which was the time period used for the toxicity studies.

Generally, over 48 h, S AgNP released more Ag than L AgNP. For the smaller AgNPs, the soluble $\mathrm{Ag}$ concentration averaged $0.95 \pm 0.05 \mu \mathrm{g} / \mathrm{L}$ and $0.99 \pm 0.11 \mu \mathrm{g} / \mathrm{L}$ at the low and high AgNP concentration, respectively. Concentrations of soluble $\mathrm{Ag}$ released from the larger AgNPs averaged $0.59 \pm 0.09 \mu \mathrm{g} / \mathrm{L}$ and $0.66 \pm 0.11 \mu \mathrm{g} / \mathrm{L}$ at the low and high AgNP concentration, respectively.

\subsection{Acute toxicity of AgNP to D. magna}

In the acute exposure of D. magna to AgNPs of two sizes, $100 \%$ mortality was observed at the highest concentration of $500 \mu \mathrm{g} / \mathrm{L}$ already after $12 \mathrm{~h}$ ( $n=20$ per treatment in 4 replicates). After $48 \mathrm{~h}$, all replicate tests reached $100 \%$ mortality at $100 \mu \mathrm{g} / \mathrm{L}$ of S AgNP and L AgNP. No mortality due to AgNP exposure was observed below $10 \mu \mathrm{g} / \mathrm{L}$ for either the smaller or larger AgNPs. All individuals survived well in the controls and no deleterious effects of PVP were observed over the $48 \mathrm{~h}$ test period.

Therefore, toxicity due to the PVP capping agent in these experiments can be ruled out.

\subsection{Metabolomics responses of D. magna to AgNP}

Representative ${ }^{1} \mathrm{H}$ NMR spectra of $D$. magna extracts from control and AgNP-exposed groups are shown in Fig. S2 

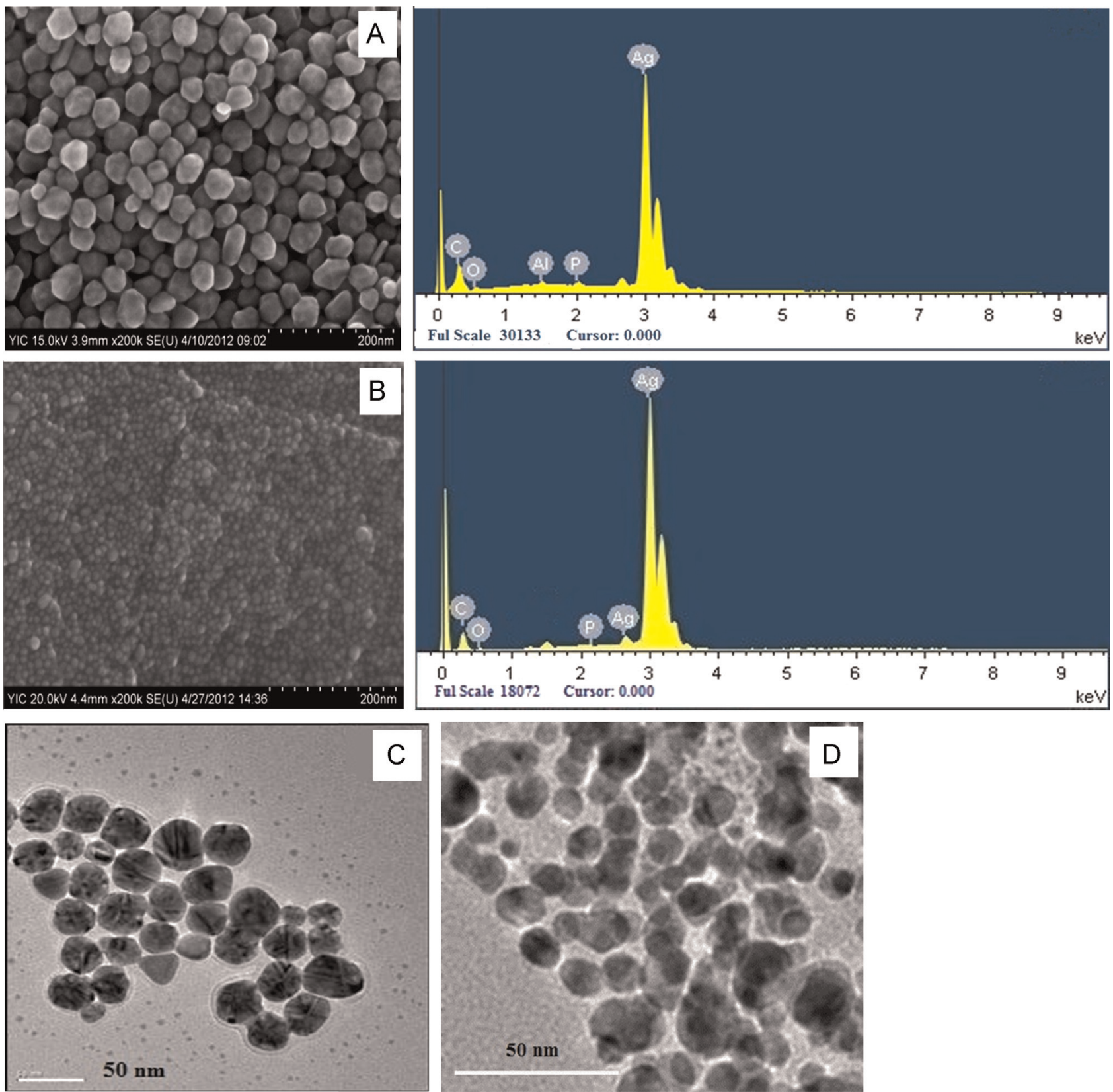

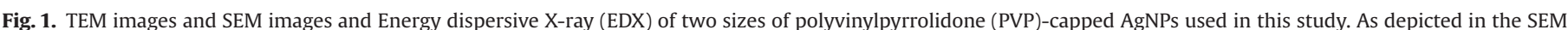

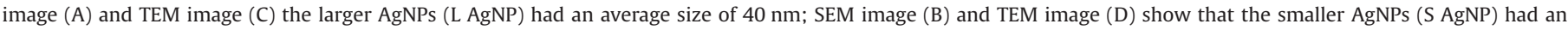
average size of $10 \mathrm{~nm}$. EDX of these NPs showed elemental Ag composition (at the right-hand side of A and B).

(Supplementary information). All the detectable metabolites in ${ }^{1} \mathrm{H}$ NMR spectra are labeled in Fig. S2 (Supplementary information). Several classes of metabolites were identified including amino acids (leucine, isoleucine, valine, alanine, glutamate, lysine, glycine, etc.), organic acids (lactate, succinate and formate) and energy storage compounds (ATP and glucose).

PCA was initially performed on the control and AgNP treatments with different sizes, respectively (Fig. 2A and B). Significant $(P<0.05)$ separations between control and AgNP-exposed groups were found along the PC1 axis. In addition, PCA resulted in significant separations between the two AgNP concentrations ( 2 and $10 \mu \mathrm{g} \mathrm{L}^{-1}$ ) for both particle sizes (10 and $40 \mathrm{~nm}$ ), respectively. The scores plots and corresponding loading plots for the pair-wise discrimination between control and AgNP-exposed groups obtained by further O-PLS-DA analysis are shown in Fig. 3. The O-PLS-DA coefficients of metabolites from the exposure to the low concentration $(2 \mu \mathrm{g} / \mathrm{L})$ of $\mathrm{S}$ AgNP $(10 \mathrm{~nm})$ revealed higher concentrations of lactate and phosphocholine than in the control group. Meanwhile, the levels of 3-hydroxybutyrate (3-HB), arginine, lysine and choline were lower than in the control group. At $10 \mu \mathrm{g} / \mathrm{L}, \mathrm{S}$ AgNP (10 nm) induced similar metabolic responses including increased lactate and phosphocholine and decreased 3-HB, arginine, lysine and choline levels. However, the concentrations of some other amino acids, including glutamate, glutamine, tyrosine and histidine, were significantly decreased. For the treatments with L AgNP (40 nm), the low concentration $(2 \mu \mathrm{g} / \mathrm{L})$ produced a very similar metabolic profile to that induced by the low concentration of the S AgNP in D. magna. Besides the similar metabolic responses including increased lactate and phosphocholine and decreased 3-HB, arginine, lysine and choline levels, two uniquely increased amino acids, leucine and glycine, were discovered at the high concentration $(10 \mu \mathrm{g} / \mathrm{L})$ of the L AgNP-treated group. 
A

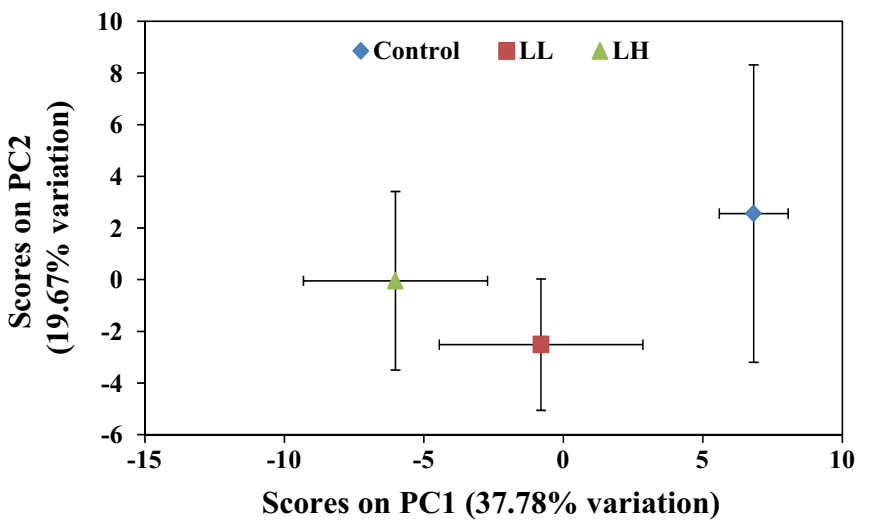

B

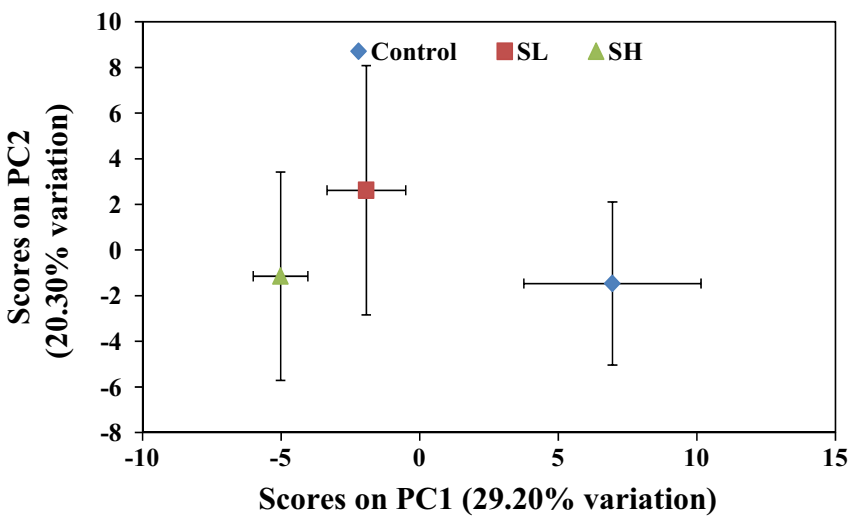

Fig. 2. Mean principal component analysis (PCA) scores plot of PC1 versus PC2 for ${ }^{1} \mathrm{H}$ NMR spectra of tissue extracts from Daphnia magna from control and AgNP exposures with two sizes, $40 \mathrm{~nm}$ (A) and $10 \mathrm{~nm}$ (B). The classes are presented as mean \pm standard deviation (as associated standard error) of PC scores for each group of $D$. magna samples. Abbreviations: LL, large size $(40 \mathrm{~nm})$ with a low concentration $(2 \mu \mathrm{g} / \mathrm{L}) ; \mathrm{LH}$, large size $(40 \mathrm{~nm})$ with a high concentrations $(10 \mu \mathrm{g} / \mathrm{L}) ; \mathrm{SL}$, small size $(10 \mathrm{~nm})$ with a low concentration $(2 \mu \mathrm{g} / \mathrm{L}) ; \mathrm{SH}$, large size $(40 \mathrm{~nm})$ with a high concentration $(10 \mu \mathrm{g} / \mathrm{L})$.

\subsection{Metabolomics responses of D. magna to $\mathrm{Ag}^{+}$}

Based on the concentrations $(\sim 0.5-1.0 \mu \mathrm{g} / \mathrm{L})$ of $\mathrm{Ag}^{+}$released from AgNPs, we designed an experiment in which $D$. magna were exposed to $\mathrm{Ag}^{+}$at 0.5 and $1.0 \mu \mathrm{g} / \mathrm{L}$, to explore the metabolic responses related to $\mathrm{Ag}^{+}$-induced toxicity. PCA was conducted on the ${ }^{1} \mathrm{H}$ NMR spectral data from $\mathrm{Ag}^{+}$-exposed $D$. magna, resulting in significant $(P<0.01)$ separations between control and $\mathrm{Ag}^{+}$-exposed groups (Fig. S3, Supplementary information). However, no significant separation was found between the two $\mathrm{Ag}^{+}$-exposed groups.

In further analysis using O-PLS-DA, scores plots and loading plots for the pair-wise discrimination between control and $\mathrm{Ag}^{+}$ exposures were obtained as shown in Fig. 4. The O-PLS-DA coefficients of metabolites from both concentrations $(0.5$ and $1.0 \mu \mathrm{g} / \mathrm{L})$ of $\mathrm{Ag}^{+}$exhibited metabolic responses similar to those of all AgNPexposed $D$. magna, including elevated phosphocholine and depleted 3-HB, arginine and lysine levels. However, glucose was significantly decreased upon $\mathrm{Ag}^{+}$exposure, which was not observed in the AgNP-exposed animals. In addition, the common elevation of lactate in AgNP-exposed daphnids was not found in both $\mathrm{Ag}^{+}$-exposed groups.

\section{Discussion}

Characterization of the AgNPs was performed both in the same medium as used in the toxicity test and in de-ionized water. A great modification of the zeta potential and the hydrodynamic diameter was observed at the exposure conditions. This was most likely due to the high ionic strength of the RW (Lok et al., 2007; Luoma 2008), which resulted in the aggregation of nanoparticles, reduced the effective surface area, and finally limited the release of soluble Ag. Despite some aggregation in the RW, the average size of the NP agglomerates remained below $100 \mathrm{~nm}$ under these conditions and remained stable for at least $48 \mathrm{~h}$, which was the time period used for the toxicity studies. It has been reported that AgNPs continuously release $\mathrm{Ag}^{+}$due to oxidation in the presence of $\mathrm{O}_{2}$ and $\mathrm{H}^{+}$(Liu and Hurt, 2010). A higher extent of release of $\mathrm{Ag}^{+}$from the suspensions of $\mathrm{S}$ AgNP versus suspensions of $\mathrm{L} \mathrm{AgNP}$ could be due to the faster release of $\mathrm{Ag}^{+}$as release is directly related to the proportionately greater total surface area. Since the $S$ AgNP have a relatively high surface/volume ratio, more atoms on the surface come in contact with the oxidant, resulting in a relatively high concentration of soluble Ag. Although larger aggregates were formed, these will not inhibit the release of soluble Ag, indicating that $\mathrm{O}_{2}$ still is able to penetrate into the aggregates and oxidize $\mathrm{Ag}(0)$ into $\mathrm{Ag}(\mathrm{I})$. Our data confirmed this expectation but did not show complete particle dissolution as was observed by Liu and Hurt (2010) in air-saturated deionized water. It should however, be noted that $\mathrm{Ag}^{+}$concentrations as found in this study and even lower can cause adverse effects to common aquatic organisms (Hogstrand and Wood, 1998), with an acute EC50 for $\mathrm{AgNO}_{3}$ of $0.68 \mu \mathrm{g} \mathrm{Ag} \mathrm{L^{-1 }}$ for D. magna (Völker et al., 2013).

A global analysis using metabolomics can provide an overall impression of the effects induced by AgNPs and $\mathrm{Ag}^{+}$based on the relevant metabolic biomarkers. Due to the dissolution of $\mathrm{Ag}^{+}$from AgNPs, however, the responses of $D$. magna in AgNP solutions could be induced not only by the AgNPs but also by the $\mathrm{Ag}^{+}$released from the AgNPs. Therefore a direct comparison of the metabolic biomarkers could indicate the similarities and differences between $\mathrm{AgNP}$ and $\mathrm{Ag}^{+}$treatments, and subsequently may elucidate the toxicological effects caused by AgNPs and/or $\mathrm{Ag}^{+}$in a certain AgNP solution. It should be noted that the concentrations of $\mathrm{Ag}^{+}$released from AgNPs dynamically changed from close to zero to the final concentrations measured in the AgNP exposures (after $48 \mathrm{~h}$ ). In this study, we used the final concentrations ( $\sim 0.5$ and $1.0 \mu \mathrm{g} / \mathrm{L}$ ) of $\mathrm{Ag}^{+}$released from the AgNPs as the nominal concentrations for the $\mathrm{AgNO}_{3}$ exposures of $D$. magna.

At both $\mathrm{Ag}^{+}$concentrations $(0.5$ and $1.0 \mu \mathrm{g} / \mathrm{L})$, the metabolic responses of $D$. magna, including elevated phosphocholine and depleted 3-HB, arginine and lysine levels, were similar to those observed in the AgNP-exposed groups. This confirmed that the $\mathrm{Ag}^{+}$released from the AgNPs induced significant metabolic alterations. Kennedy et al. (2010) indicated the importance of free silver ions as a major source of toxicity of AgNPs to D. magna and Pimephales promelas. Zhao and Wang (2012) also attributed toxicity of AgNPs to daphnids mainly to the release of soluble Ag, as similar 48 h LC50s were obtained for lactate-coated AgNPs, PVPcoated AgNPs and $\mathrm{AgNO}_{3}$, when the daphnids were exposed to the soluble Ag released from AgNPs. For both nano-sized AgNP exposures, PCA resulted in significant separations between low $(2 \mu \mathrm{g} / \mathrm{L})$ and high $(10 \mu \mathrm{g} / \mathrm{L})$ exposure concentrations (Fig. 2). This observation clearly showed significant concentration-dependent metabolic responses.

In $\mathrm{Ag}^{+}$-exposed D. magna, 3-HB, arginine, lysine and glucose were depleted. 3 -HB can be formed during fatty acid oxidation and its depletion suggests a response to the decreased energy demand resulting in the decreased gluconeogenesis in organisms (Bertini et al., 2012). Therefore the decreased 3-HB level might imply a decreased energy demand in $D$. magna after exposure to $\mathrm{Ag}^{+}$, together with decreased glucose levels. As a phosphagen, phosphocholine is biosynthesized by choline kinase converting ATP and 
A

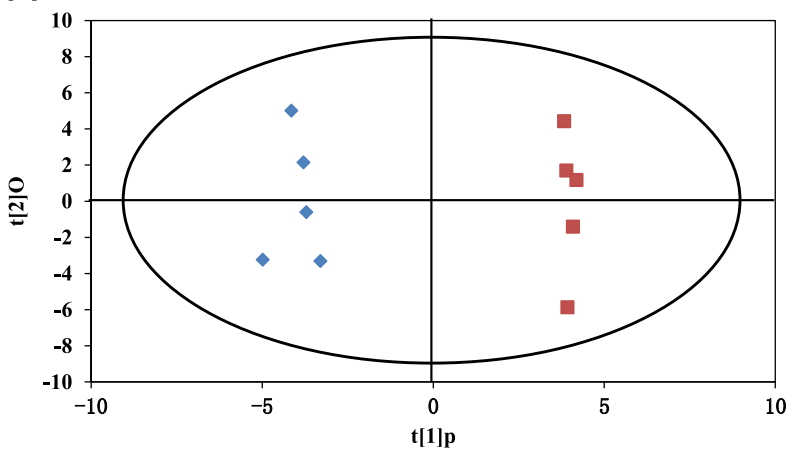

$R^{2} X=54.2 \%, Q^{2}=0.838$

C

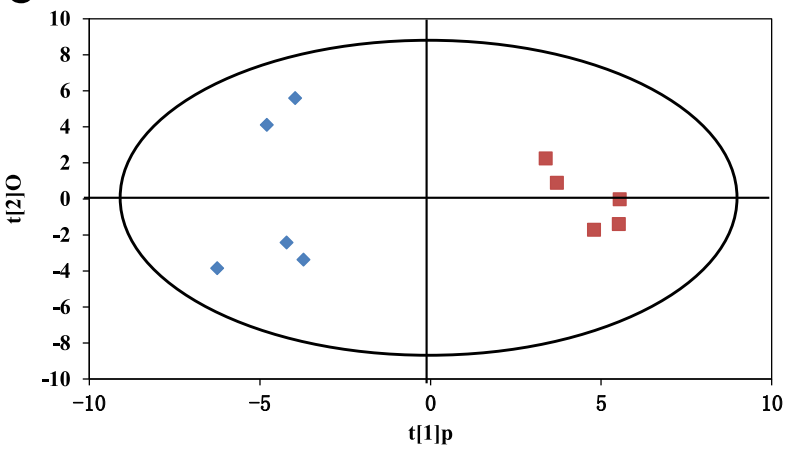

$R^{2} X=57.9 \%, Q^{2}=0.786$

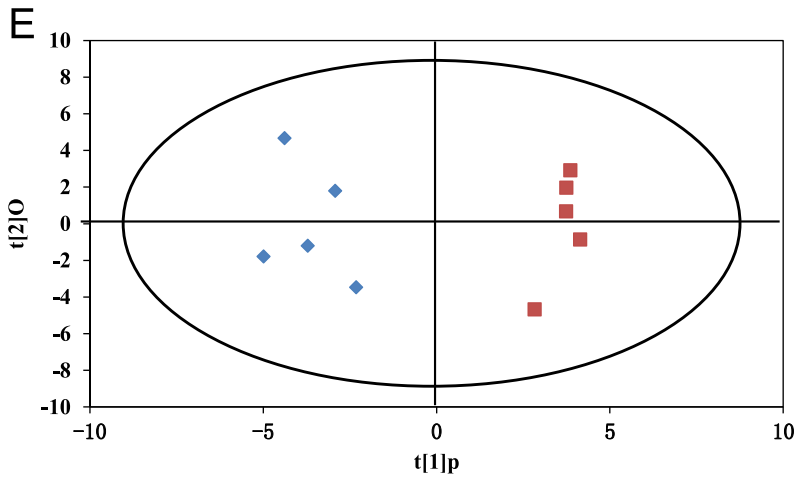

$R^{2} X=49.0 \%, Q^{2}=0.751$

G

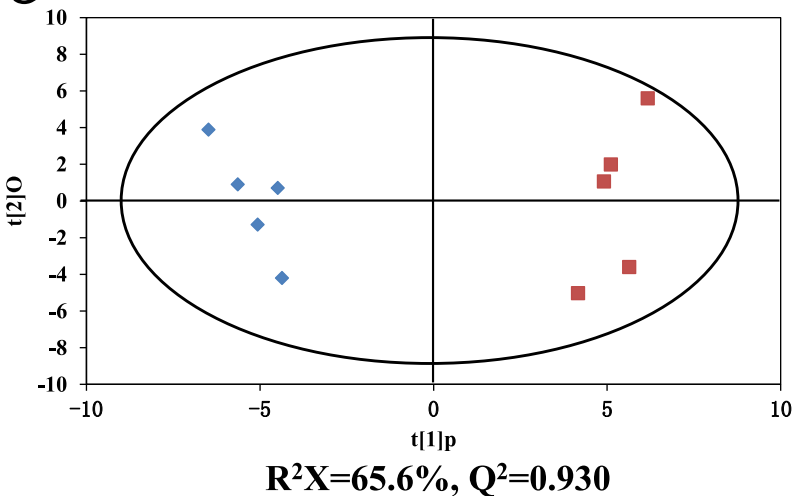

B

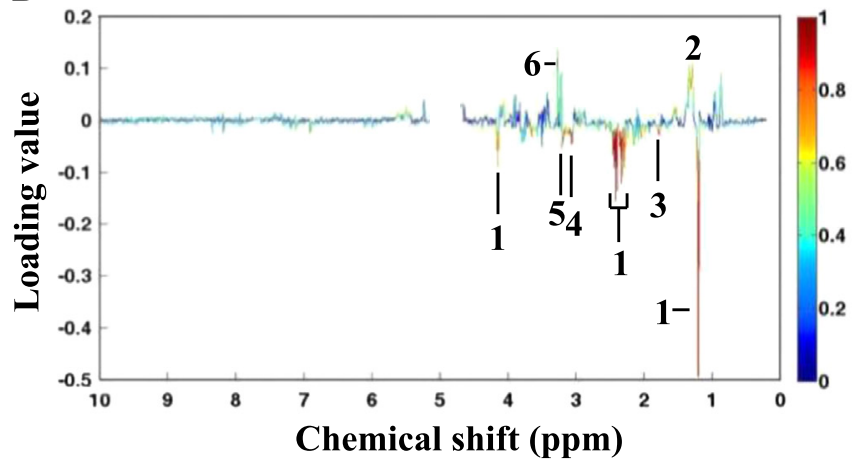

D

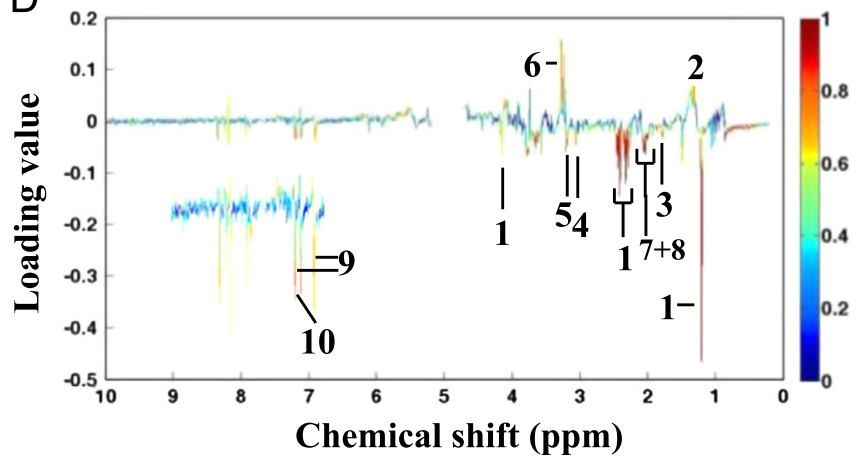

$\mathrm{F}$

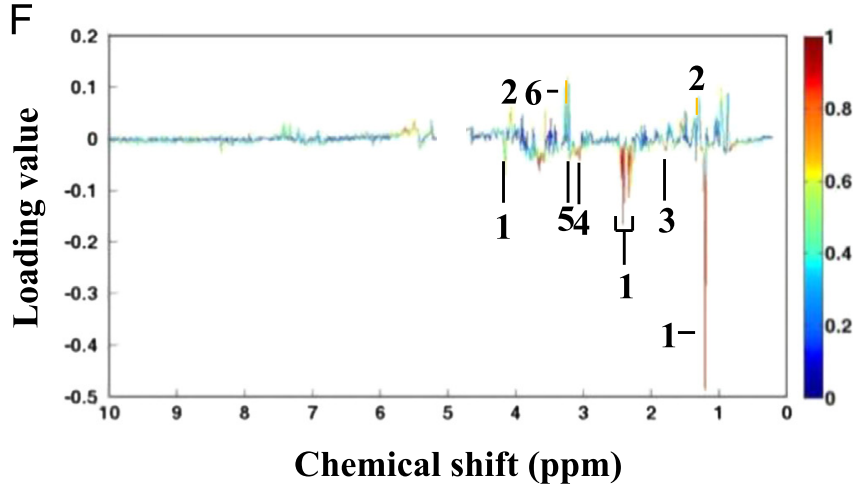

$\mathrm{H}$

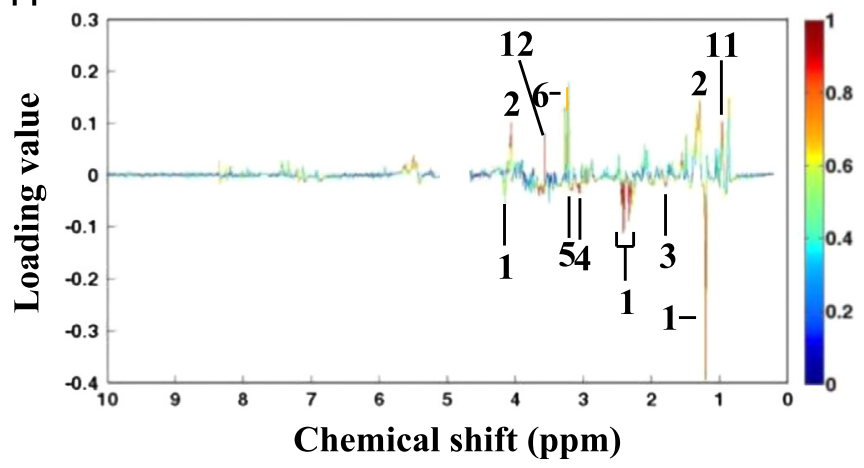

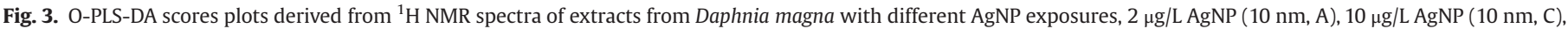

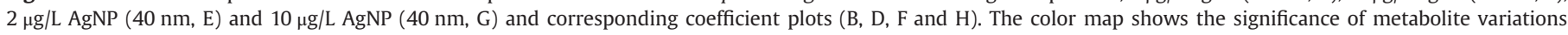

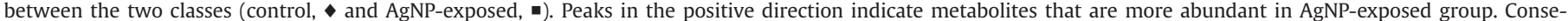

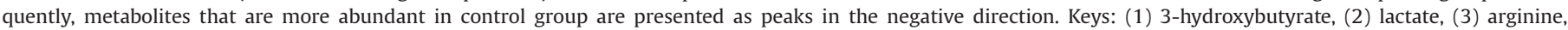
(4) lysine, (5) choline, (6) phosphocholine, (7) glutamate, (8) glutamine, (9) tyrosine, (10) histidine, (11) leucine and (12) glycine. 
A

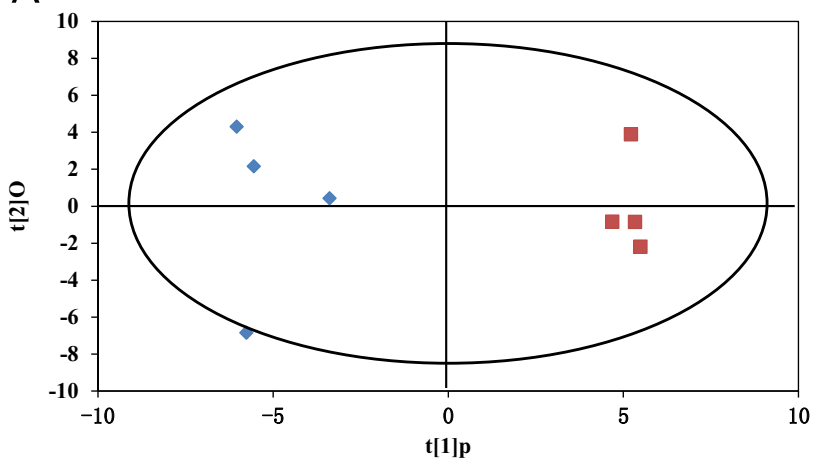

$R^{2} X=72.9 \%, Q^{2}=0.927$

C

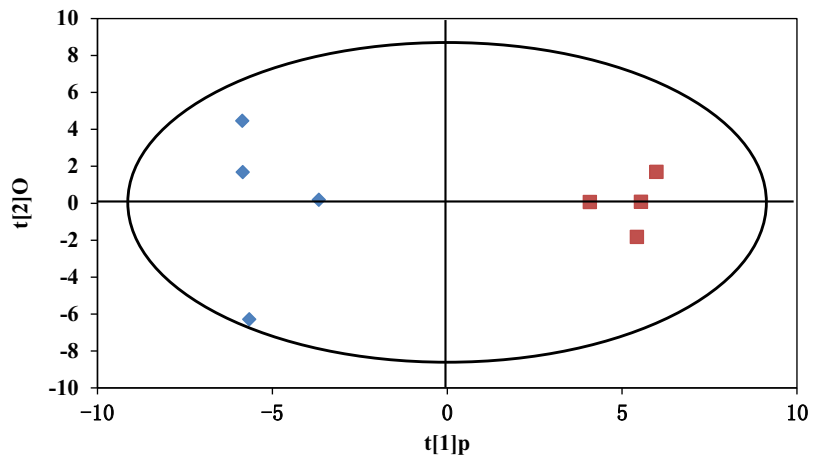

$R^{2} X=66.0 \%, Q^{2}=0.806$
B

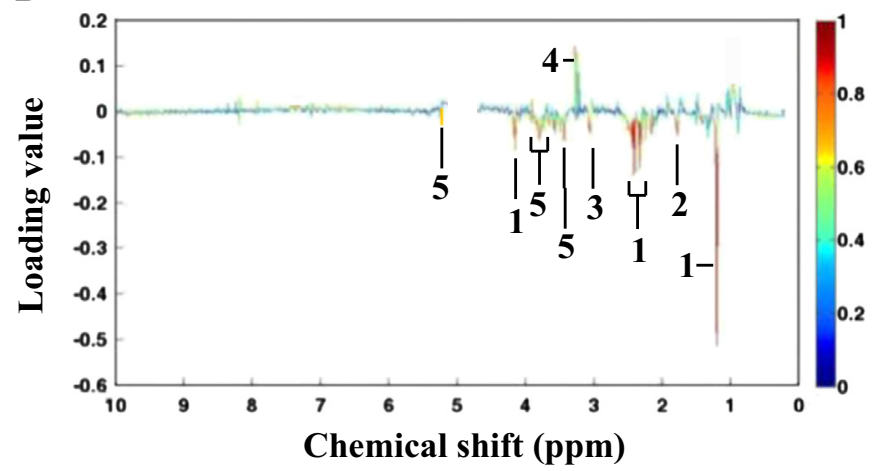

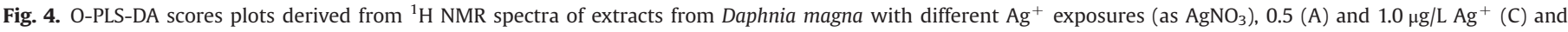

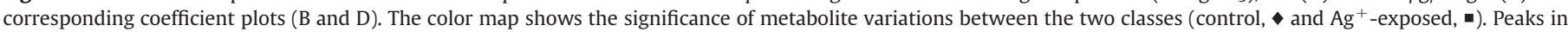

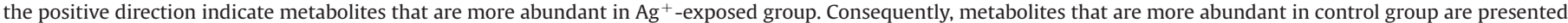
as peaks in the negative direction. Keys: (1) 3-hydroxybutyrate, (2) arginine, (3) lysine, (4) phosphocholine and (5) glucose.

choline into phosphocholine and ADP. The elevation of phosphocholine probably signaled the disturbance in energy metabolism. Decreases in amino acid (arginine and lysine) levels were observed in $\mathrm{Ag}^{+}$-exposed D. magna. In previous studies on the toxicity of $\mathrm{Cu}^{2+}$, both arginine and lysine were found to decrease together with other amino acids including tyrosine, leucine, isoleucine and tryptophan in $D$. magna. This was accounted for as a general depletion of amino acids in response to the induction of defense and repair mechanisms, such as the synthesis of stress proteins and DNA repair enzymes, towards $\mathrm{Cu}^{2+}$-induced oxidative stress (Knops et al., 2001; Smolders et al., 2005). A similar metabolic response with decreased amino acid levels was also observed in $\mathrm{Cu}^{2+}$-exposed earthworms Lumbricus rubellus (Bundy et al., 2008). Since $\mathrm{Ag}^{+}$is a metal known to induce oxidative stress in organisms (Cortese-Krott et al., 2009), the decreased amino acid (arginine and lysine) levels were indicative of the impact of $\mathrm{Ag}^{+}$ exposure in D. magna. Taken into consideration all these metabolic biomarkers, we found that $\mathrm{Ag}^{+}$exposure $(0.5$ and $1.0 \mu \mathrm{g} / \mathrm{L})$ induced disturbance of energy metabolism and oxidative stress in $D$. magna.

Compared to the metabolic profiles of $\mathrm{Ag}^{+}$-exposed D. magna, the similarly altered metabolites (3-HB, arginine, lysine and phosphocholine) in case of AgNP exposure, implied that the $\mathrm{Ag}^{+}$ released from both AgNPs induced disturbance in energy metabolism and oxidative stress. However, the levels of lactate were elevated in all AgNP-treated groups but not in the $\mathrm{Ag}^{+}$-treated groups. This observation could be caused by increased movement and perturbed breathing in AgNP exposure groups. A proposed mechanism includes direct ingestion of AgNPs and binding of nanoparticles directly to the daphnids (Gilroy et al. 2014), including potentially binding to the gas exchange apparatus of the organisms. Exposure to AgNPs can enhance anaerobic metabolism in D. magna, which has been proposed as a toxic mechanism of action related to nanomaterial exposure in a variety of organisms including D. magna (Zhang et al., 2006), but this must be confirmed by further, more targeted testing. The decrease of choline could be related to the increase of phosphocholine in all AgNPtreated groups. Interestingly, a depletion of glucose induced by $\mathrm{Ag}^{+}$was not detected in AgNP-exposed animals, probably due to the slow release of silver ions from the particles, which might alleviate the inhibition in the gluconeogenesis in $D$. magna. For the exposure to the high AgNP concentration $(10 \mu \mathrm{g} / \mathrm{L})$, the decrease of some other amino acids including glutamate, glutamine, tyrosine and histidine might imply that the S AgNP caused more severe oxidative stress in D. magna.

\section{Conclusions}

D. magna exhibited significant changes in their metabolic profile following the two size $\mathrm{AgNP}$ and $\mathrm{Ag}^{+}$exposures. Most of the metabolic biomarkers for AgNP exposure were identical to those of the $\mathrm{Ag}^{+}$-exposed groups, suggesting that the dominant effects of both AgNPs were due to released $\mathrm{Ag}^{+}$. The observed metabolic responses to the $\mathrm{Ag}^{+}$released from both the AgNPs is indicative of disturbances of energy metabolism and oxidative stress in $D$. magna. Moreover, the levels of lactate were elevated in all AgNPtreated groups but not in the $\mathrm{Ag}^{+}$-treated groups. This suggests a 
mechanism of enhancement of anaerobic metabolism being induced by AgNPs.

\section{Declaration of interest}

The authors report no conflicts of interest. The authors alone are responsible for the content and writing of the paper.

\section{Acknowledgements}

This study is supported by the National Natural Science Foundation of China (No. Y311111031). Part of the work was performed within the framework of the RIVM sponsored project "IRAN". The authors are grateful for the assistance in silver nanoparticle preparation from Dr. Liping You in Yantai Institute of Coastal Zone Research (YIC).

\section{Appendix A. Supplementary information}

Supplementary data associated with this article can be found in the online version at http://dx.doi.org/10.1016/j.ecoenv.2015.05. 005.

\section{References}

Baun, A., Hartmann, N.B., Grieger, K., Kusk, K.O., 2008. Ecotoxicity of engineered nanoparticles to aquatic invertebrates: a brief review and recommendations for future toxicity testing. Ecotoxicology 17 (5), 387-395.

Bertini, I., Cacciatore, S., Jensen, B.V., Schou, J.V., Johansen, J.S., Kruhøffer, M., Luchinat, C., Nielsen, D.L., Turano, P., 2012. Metabolomic NMR fingerprinting to identify and predict survival of patients with metastatic colorectal cancer. Cancer Res. 72 (1), 356-364.

Bilberg, K., Malte, H., Wang, T., Baatrup, E., 2010. Silver nanoparticles and silver nitrate cause respiratory stress in Eurasian perch (Perca fluviatilis). Aquat. Toxicol. 96 (2), 159-165.

Bundy, J.G., Davey, M.P., Viant, M.R., 2009. Environmental metabolomics: a critical review and future perspectives. Metabolomics 5 (1), 3-21.

Bundy, J.G., Sidhu, J.K., Rana, F., Spurgeon, D.J., Svendsen, C., Wren, J.F., Sturzenbaum, S.R., Morgan, A.J., Kille, P., 2008. Systems toxicology approach identifies coordinated metabolic responses to copper in a terrestrial non-model invertebrate the earthworm Lumbricus rubellus. BMC Biol 6, 25.

Chae, Y.J., Chi, P.C.H., Lee, J., Bae, E., Yi, J., Gu, M.B., 2009. Evaluation of the toxic impact of silver nanoparticles on. Japanese medaka (Oryzias latipes). Aquat. Toxicol. 94 (4), 320-327.

Cortese-Krott, M.M., Münchow, M., Pirevc, E., Heßnerc, F., Bozkurtb, A., Uciechowskid, P., Palluab, N., Krönckec, K.-D., Suschekb, C.V., 2009. Silver ions induce oxidative stress and intracellular zinc release in human skin fibroblasts. Free Radic. Biol. Med. 47 (11), 1570-1577.

Fan, W.M.T., 1996. Metabolite profiling by one- and two-dimensional NMR analysis of complex mixtures. Prog. Nucl. Magn. Reson. Spectrosc. 28 (2), 161-219.

Feng, J., Li, J., Wu, H., Chen, Z., 2013. Metabolic responses of HeLa cells to silica nanoparticles by NMR-based metabolomic analyses. Metabolomics 9 (4), 874-886.

Feng, J., Zhao, J., Hao., F., Chen, C., Bhakoo, K., Tang, H., 2011. NMR-based metabonomics analyses of the effects of ultrasmall superparamagnetic particles of iron oxide (USPIO) on macrophage metabolism. J. Nanopart. Res. 13 (5), 2049-2062.

Gilroy, K.D., Neretina, S., Sanders, R.W., 2014. Behavior of gold nanoparticles in an experimental algal-zooplankton food chain. J. Nanopart. Res. 16 (5), 2414

Griffitt, R.J., Luo, J., Gao, J., Bonzongo, J.C., Barber, D.S., 2008. Effects of particle composition and species on toxicity of metallic nanomaterials in aquatic organisms. Environ. Toxicol. Chem. 27 (9), 1972-1978.

Hadrup, N., Lam, H.R., Loeschner, K., Mortensen, A., Larsen, E.H., Frandsen, H., 2012. Nanoparticulate silver increases uric acid and allantoin excretion in rats, as identified by metabolomics. J. Appl. Toxicol. 32 (11), 929-933.

Hogstrand, C. Wood, C.M., 1998. Toward a better understanding of the bioavailability, physiology and toxicity of silver in fish: implications for water quality criteria. Environ. Toxicol. Chem. 17 (4), 547-561.

Kennedy, A., Hull, M., Bednar, A.J., Goss, J., Gunter, J., Bouldin, J., Vikesland, P., Steevens, J., 2010. Fractionating nanosilver: importance for determining toxicity to aquatic test organisms. Environ. Sci. Technol. 44 (24), 9571-9577.

Kim, J., Kim, S., Lee, S., 2011. Differentiation of the toxicities of silver nanoparticles and silver ions to the Japanese medaka (Oryzias latipes) and the cladoceran Daphnia magna. Nanotoxicology 5 (2), 208-214.

Knops, M., Altenburger, R., Segner, H., 2001. Alterations of physiological energetics, growth and reproduction of Daphnia magna under toxicant stress. Aquat. Toxicol. 53 (2), 79-90.

Laban, G., Nies, L.F., Turco, R.F., Bickham, J.W., Sepúlveda, M.S., 2010. The effects of silver nanoparticles on fathead minnow (Pimephales promelas) embryos. Ecotoxicology 19 (1), 185-195.

Li, T., Albee, B., Alemayehu, M., Diaz, R., Ingham, L., Kamal, S., Rodriguez, M., Whaley Bishnoi, S., 2010. Comparative toxicity study of Ag, Au, and Ag-Au bimetallic nanoparticles on Daphnia magna. Anal. Bioanal. Chem. 398 (2), 689-700.

Lin, C.Y. Viant, M.R., Tjeerdema, R.S., 2006. Metabolomics: methodologies and applications in the environmental sciences. J. Pestic. Sci. 31 (3), 245-251.

Liu, J.F., Chao, J.B., Liu, R., Tan, Z.Q., Yin, Y.G., Wu, Y., Jiang, G.B., 2009. Cloud point extraction as an advantageous preconcentration approach for analysis of trace silver nanoparticles in environmental waters. Anal. Chem. 81 (15), 6496-6502.

Liu, J.Y., Hurt, R.H., 2010. Ion release kinetics and particle persistence in aqueous nano-silver colloids. Environ. Sci. Technol. 44 (6), 2169-2175.

Liu, X., Zhang, L., You, L., Cong, M., Zhao, J., Wu, H., Li, C.H., Liu, D.Y., Yu, J.B., 2011 Toxicological responses to acute mercury exposure for three species of Manila clam Ruditapes philippinarum by NMR-based metabolomics. Environ. Toxicol. Pharmacol. 31 (2), 323-332.

Lok, C.N., Ho, C.M., Chen, R., He, Q.Y., Yu, W.Y., Sun, H., Tam, P.H., Chiu, J.F., Che, C.M., 2007. Silver nanoparticles: Partial oxidation and antibacterial activities. J. Biol. Inorg. Chem. 12 (4), 527-534.

Luoma, S.N., 2008. Silver Nanotechnologies and the Environment: Old Problems or New challenges? In Project on Emerging Nanotechnologies. PEW Charitable Trusts, Washington, DC.

Massarsky, A., Dupuis, L., Taylor, J., Eisa-Beygi, S., Strek, L., Trudeau, V.L., Moon, T.W. 2013. Assessment of nanosilver toxicity during zebrafish (Danio rerio) development. Chemosphere 92 (1), 59-66.

Meyer, J.N., Lord, C.A., Yang, X.Y.Y., Turner, E.A., Badireddy, A.R., Marinakos, S.M., Chilkoti, A., Wiesner, M.R. Auffan, M., 2010. Intracellular uptake and associated toxicity of silver nanoparticles in Caenorhabditis elegans. Aquat. Toxicol. 100 (2), 140-150.

Navarro, E., Piccapietra, F., Wagner, B., Marconi, F., Kaegi, R., Odzak, N., Sigg, L. Behra, R., 2008. Toxicity of silver nanoparticles to Chlamydomonas reinhardtii. Environ. Sci. Technol. 42 (23), 8959-8964.

OECD, 2004. Guidelines for the testing of chemicals: Daphnia sp., acute Immobilization Test, test no. 202. Organization for Economic Cooperation and Development. Paris, France.

Parsons, H.M., Ludwig, C., Gunther, U.L., Viant, M., 2007. Improved classification accuracy in 1- and 2-dimensional NMR metabolomics data using the variance stabilising generalised logarithm transformation. BMC Bioinformatics 8, 234.

Smolders, R., Baillieul, M., Blust, R., 2005. Relationship between the energy status of Daphnia magna and its sensitivity to environmental stress. Aquat. Toxicol. 73 (2), 155-170.

Viant, M.R., Rosenblum, E.S., Tjeerdema, R.S., 2003. NMR-based metabolomics: A powerful approach for characterizing the effects of environmental stressors on organism health. Environ. Sci. Technol. 37 (21), 4982-4989.

Völker, C., Boedicker, C., Daubenthaler, J., Oetken, M., Oehlmann, J., 2013. Comparative toxicity assessment of nanosilver on three Daphnia species in acute, chronic and multi-generation experiments. PLoS One 8 (10), e75026.

Whitfield Aslund, M.L. McShane, H. Simpson, M.J. Simpson, A.J., Whalen, J.K., Hendershot, W.H., Sunahara, G.I., 2012. Earthworm sublethal responses to titanium dioxide nanomaterial in soil detected by ${ }^{1} \mathrm{H}$ NMR metabolomics. Environ. Sci. Technol. 46 (2), 1111-1118.

Wu, H., Southam, A.D., Hines, A., Viant, M.R., 2008. High throughput tissue extraction protocol for NMR and mass spectrometry based metabolomics. Anal. Biochem. 372 (2), 204-212.

Zhang, X., Wu, H., Liao, P., Li, X., Ni, J., Pei, F., 2006. NMR-based metabonomic study on the subacute toxicity of aristolochic acid in rats. Food Chem. Toxicol. 44 (7), 1006-1014.

Zhao, C.M., Wang, W.X., 2012. Size-dependent uptake of silver nanoparticles in Daphnia magna. Environ. Sci. Technol. 46 (20), 11345-11351. 